\title{
Characteristics of the initial seizure in familial febrile seizures
}

\begin{abstract}
$M$ van Stuijvenberg, E van Beijeren, $N$ H Wils, G Derksen-Lubsen, C M van Duijn, H A Moll
\end{abstract}

\begin{abstract}
Complex seizure characteristics in patients with a positive family history were studied to define familial phenotype subgroups of febrile seizures. A total of 51 children with one or more affected first degree relatives and 177 without an affected first degree relative were compared for history of complex characteristics of the initial febrile seizure. No difference was found in the frequency of febrile status epilepticus $(O R=1.1(95 \%$ confidence interval (CI) 0.3 to 4.3 )), multiple type $(\mathrm{OR}=0.6(\mathrm{CI} 0.3$ to 1.2$))$, and focal characteristics $(\mathrm{OR}=0.4(\mathrm{CI} 0.2$ to 1.2$)$ ). The presence of any complex characteristic $(\mathrm{OR}=0.5$ (CI 0.3 to 1.0$)$ ) was higher in those without an affected first degree relative, although differences did not reach significance. The familial type of febrile seizures is not associated with complex characteristics of the initial febrile seizure. Complex seizure characteristics are unlikely to help in discriminating phenotype subgroups for genetic studies of febrile seizures.

(Arch Dis Child 1999;80:178-180)
\end{abstract}

Keywords: febrile seizures; genetics; epilepsy

Department of Paediatrics, University

Hospital, Leiden, the

Netherlands

E van Beijeren

Department of

Paediatrics, Juliana

Children's Hospital,

Den Haag, the

Netherlands

G Derksen-Lubsen

Department of

Epidemiology and

Biostatistics, Erasmus

University, Rotterdam,

the Netherlands

$C M$ van Duijn

Department of

Paediatrics, Sophia

Children's Hospital,

Rotterdam, the

Netherlands

H A Moll

Correspondence to:

Dr H A Moll, Department of

Paediatrics, Room Sp 1543 ,

Sophia Children's Hospital,

Dr Molewaterplein 60, 3015

GJ Rotterdam, Netherlands.

Accepted 8 September 1998 zures will be the dissection of the heterogeneous group of children with febrile seizures into subgroups of patients with a more homogeneous phenotype.

We examined whether familial febrile seizures can be characterised by complex seizure characteristics. Characteristics of the initial febrile seizure were compared between children with one or more affected first degree relatives and children without an affected first degree relative.

\section{Patients and methods}

Children who consecutively visited the Sophia Children's Hospital in Rotterdam or the Juliana Children's Hospital in the Hague between 1994 and 1996 with a febrile seizure were considered for inclusion. They were selected from the prospective febrile seizure registration, established at the paediatric department of the Sophia Children's Hospital since 1988. The paediatric department of the Juliana Children's Hospital joined this registration in 1994. All patients in this registration visited the febrile seizure outpatient clinic within two to four weeks after a febrile seizure. As well as routine patient follow up, baseline characteristics of the patients were registered, including the family history. A febrile seizure was defined according to the National Institute of Health consensus for febrile seizures, excluding any seizure caused by an underlying abnormality and excluding any seizure occurring after a previous non-febrile seizure ${ }^{10}$ Children with an unknown family history were excluded from the analysis. Children of nonwhite origin were excluded to obtain a genetically homogeneous study sample.

Children were included in the case group if they had one or more first degree relatives affected with febrile seizures: parents, brothers, and sisters. The referent group consisted of all children who had no first degree relatives affected by febrile seizures.

\section{DATA COLLECTION}

The following data were studied: baseline characteristics, including age at the initial febrile seizure (age at onset); sex; and the presence of complex characteristics of the initial febrile seizure. A seizure was defined as complex if it lasted 30 minutes or longer (febrile status epilepticus) and/or if the seizure recurred within 24 hours (multiple type) and/or if the seizure had a focal onset or a postictal Todd's paresis of facial muscles or limbs (focal characteristics). ${ }^{1112}$ Seizure characteristics were determined based on the history given by the child's parents or other witnesses of the seizure, or on the documentation in the 
Table 1 Baseline characteristics and characteristics of the initial febrile seizure

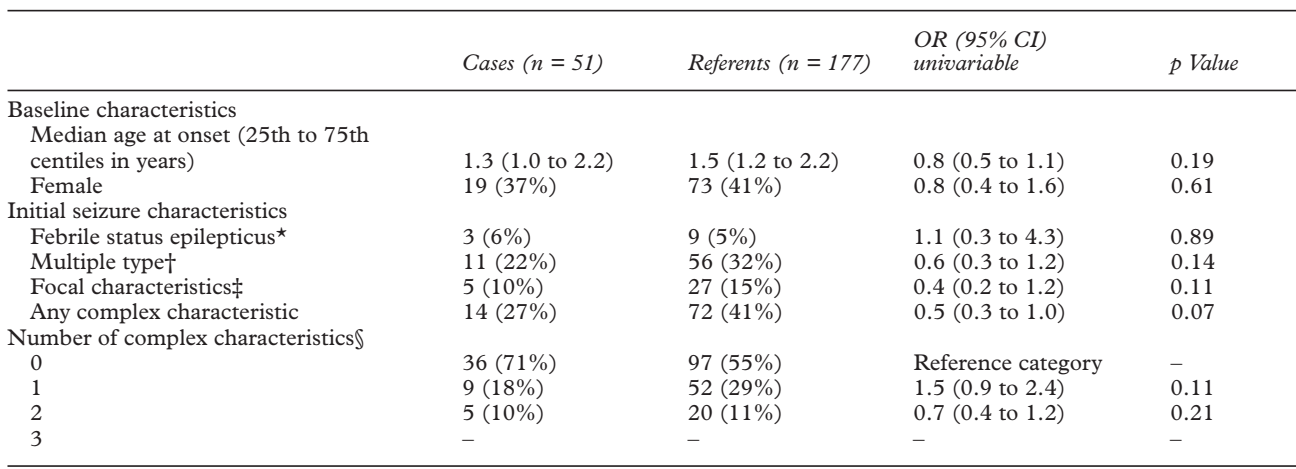

*Missing values: $3(6 \%) v 19(11 \%)$

†Missing values: $3(6 \%) v 14(8 \%)$.

$\neq$ Missing values: $12(23 \%) v 72(41 \%)$

Analysed as a continuous variable: $\mathrm{OR}=0.7$ (0.4 to 1.1$) \mathrm{p}=0.14$.

patient's chart. If characteristics of the initial seizure were unknown, they were considered missing values.

For the analysis, second and further degree relatives were not taken into account. Siblings and parents with epileptic disorders, but without a history of febrile seizures, were considered not affected.

\section{STATISTICAL ANALYSIS}

The relation between the characteristics of the initial febrile seizure (febrile status epilepticus, multiple type, and focal characteristics) in the case group and the control group was estimated using logistic regression analysis. Odds ratios (ORs) were used as the measure of association; associations were statistically significant $(p<0.05)$ if the $95 \%$ confidence interval (CI) of the OR did not include the value 1 . SPSS 6.0 for Windows was used for the analysis.

\section{Results}

Of all 478 children who had visited one of the two participating hospitals because of a febrile seizure between 1994 and 1996, 240 children were of non-white origin. For 10 of the remaining eligible children, the family history was unknown; thus, 228 children entered the study.

Of these 228, $51(22 \%)$ children had at least one affected first degree relative and were included in the case group: $14(27 \%)$ of the 51 children had one or more affected siblings, 31 $(61 \%)$ had one affected parent, and six $(12 \%)$ had one affected parent and one or more affected siblings. The referent group comprised $177(78 \%)$ of 228 children without a first degree relative affected by febrile seizures.

Table 1 shows the baseline characteristics and the characteristics of the initial febrile seizure of the cases and the referents. Using logistic regression, no difference was shown in age at onset or sex. Febrile status epilepticus did not differ between the two groups; multiple type and focal characteristics were counted more frequently in the referent group; the presence of any complex characteristic was observed more frequently in the referent group, although differences did not reach significance $(p=0.07)$. The number of complex characteristics for each seizure did not show a difference between the case and the referent group.

\section{Discussion}

This study shows that the familial type of febrile seizures is unlikely to be associated with complex characteristics of the initial seizure. Thus, defining a phenotype subgroup associated with complex seizure characteristics may be unhelpful in genetic studies to localise genes involved in febrile seizures. The results suggested even higher frequencies of complex seizure characteristics in the referent group, although differences were not statistically significant. These results are supported by a study that showed no difference in "seizure severity" in familial versus non-familial febrile seizures. In the previous study, no further specification was made on how seizure severity was defined and whether complex seizure characteristics were considered. ${ }^{13}$ Another study of familial febrile seizures suggested that the proportion of complex febrile seizures was higher among multicase (familial) compared with single case (non-familial) probands; statistical significance, however, was not reached. ${ }^{6}$ The results of the present study are in accordance with these previous studies.

A large population based study of 2609 relatives (parents, siblings, children, nieces, nephews, and half-siblings) of 421 children with febrile seizures investigated the risk for the development of febrile seizures in siblings. As well as frequent recurrent seizures, complex seizures in the proband were associated with an increased risk of febrile seizures in siblings, which may suggest that complex seizures are associated with a familial predisposition. ${ }^{1}$ In a recent study of 398 first degree relatives of 129 children with febrile seizures, the risk of febrile seizures in siblings was increased when the proband had had recurrent febrile seizures. ${ }^{14}$ Unfortunately, complex characteristics were not considered in the analysis. ${ }^{14}$ These two studies used a different strategy to investigate phenotype subgroups which may be helpful to study the hereditary basis of febrile seizures. Their approach has been based on studying a 
sample of the relatives of the proband. Thus, their results can not easily be compared with ours. Both previous studies ${ }^{114}$ suggested that recurrent febrile seizures might be characteristic of hereditary febrile seizures, and one of them $^{1}$ also suggested that complex seizure characteristics might help to delineate a subgroup worthy of genetic study.

The limitation of the present study is the high number of missing values for focal seizure characteristics. This is likely to be due to the relative difficulty of recognising a focal seizure feature. In a study assessing the accuracy of the classification of complex characteristics of febrile seizures, experienced paediatric neurologists most often showed disagreement about assessment of focal seizure characteristics. They disagreed less about multiple and prolonged characteristics. ${ }^{11}$

In conclusion, we provided no evidence for an association between familial febrile seizures and complex characteristics of the initial febrile seizure of the proband. This does not exclude the existence of other clinical features - that is, characteristics of the seizure which are specific for patients with familial forms of febrile seizures. Complex febrile seizure characteristics, however, are unlikely to help in discriminating phenotype subgroups for genetic studies of febrile seizures.
1 Hauser WA, Annegers JF, Anderson VE, Kurland LT. The risk of seizure disorders among relatives of children with risk of seizure disorders among relatives of childre

2 Offringa M, Bossuyt PMM, Lubsen J, et al. Risk factors for seizure recurrence in children with febrile seizures: a pooled analysis of individual patient data of five studies. $\mathcal{F}$ Pediatr 1994;124:574-84.

3 Berg AT, Shinnar S, Hauser WA, et al. A prospective study of recurrent febrile seizures. N Engl f Med 1992;327:1122-7.

4 Esch A van, Steyerberg EW, Berger MY, Offringa M, Derksen-Lubsen G, Habbema JDF. Family history and recurrence of febrile seizures. Arch Dis Child 1994;70:3959 .

5 Rantala H, Uhari M. Risk factors for recurrences of febrile seizures. Acta Neurol Scand 1994;90:207-10.

6 Johnson WG, Kugler SL, Stenroos ES, et al. Pedigree analysis in families with febrile seizures. Am $\mathcal{F}$ Med Genet 1996; 61:345-52.

7 Doose H, Maurer A. Seizure risk in offspring of individuals with a history of febrile convulsions. Eur $\mathcal{F}$ Pediatr with a history

8 Rich SS, Annegers JF, Hauser WA, Anderson VE. Complex segregation analysis of febrile convulsions. Am $\mathcal{F}$ Hum Genet 1987;41:249-57.

9 Berg AT, Shinnar S, Hauser WA, Leventhal JM. Predictors of recurrent febrile seizures: a meta-analytic review. $\mathcal{F}$ Pediatr 1990;116:329-37.

10 National Institutes of Health consensus statement. Febrile seizures. Pediatrics 1980;66:207-10.

11 Berg AT, Steinschneider M, Kang H, Shinnar S. Classification of complex features of febrile seizures: interrater agreement. Epilepsia 1992;33:661-6.

12 Commission on Epidemiology and Prognosis of the International League against Epilepsy. Guidelines for epidemiologic studies on epilepsy. Epilepsia 1993;34:5926.

13 Frantzen E, Lennox-Buchthal M, Nygaard A, Stene J. A genetic study of febrile convulsions. Neurology 1970;20: 909-17.

14 Esch A van, Steyerberg EW, Duijn van CM, Offringa $M$, Derksen-Lubsen G, Steensel van-Moll HA. Prediction of febrile seizures in siblings: a practical approach. Eur $\mathcal{F}$ Pediatr 1998;157:340-4. 